\title{
Wada basins and qualitative unpredictability in ecological models: a graphical interpretation
}

\author{
John Vandermeer ${ }^{k}$ \\ Department of Ecology and Evolutionary Biology, University of Michigan, Ann Arbor, MI 480109, USA
}

Received 14 January 2003; received in revised form 12 September 2003; accepted 27 October 2003

\begin{abstract}
The capability of ecological models to make precise predictions was questioned with the discovery of chaos. Here it is shown that an alternative form of unpredictability is associated with some nonlinear models. The notion of a Wada basin, in which three or more basins share complexly interdigitated boundaries, represents this new form of unpredictability. It signifies that a single point seemingly balanced between two basins of attraction may in fact just as easily travel to a third, seemingly unconnected, basin. A circle map approximation to coupled predator prey pairs is used to demonstrate certain qualitative properties associated with the equilibria within the basins. Most important is the demonstration that the ultimate basin in which a trajectory comes to lie may not be confidently predicted from detailed knowledge of the point of initiation. Furthermore, when Wada basin boundaries become large, a small amount of stochastic forcing may create chaos-like behavior (though not formally chaotic) in a system that has, mathematically, only stable equilbria. The generation of Wada basins is discussed, using piecewise linear maps.
\end{abstract}

(C) 2004 Elsevier B.V. All rights reserved.

Keywords: Wada basins; Ecological model; Unpredictability

\section{Introduction}

The standard goal of ecological models is to predict the behavior of ecosystems, either in a precise engineering-like fashion or as qualitative generalizations (Levins. 1966: May. 1973) Ambitious simulator models are usually able to predict the actual state of an ecosystem only approximately, and it has been much more common to seek qualitative predictions - on the one hand "how many individuals will be in the population next year?" and on the other "will the population persist or go extinct?" For example, in predicting the population density of an agricultural pest, practitioners might wish to predict exact pop-

\footnotetext{
* Tel.: +1-734-764-1446; fax: +1-734-763-0544.

E-mail address: jvander@umich.edu (J. Vandermeer).
}

ulation densities, but usually are content if they are able to predict whether or not the population exceeds the economic threshold. Similarly, conservation biologists are mainly concerned with whether a species will persist or become extinct, not with estimates of its exact population density.

The ability of ecological models to make precise predictions was severely compromised with the discovery of chaos (May. 1976: Hastings et al. 1993): sensitive dependence on initial conditions means that precise prediction of particular states is effectively impossible. A similar warning of inherent unpredictability was issued later (May. 1977) for a different but ultimately related reason: If alternate states exist, for any trajectory initiated at the boundary of the basins of attraction of those alternate states it would be difficult to predict which state would ultimately capture 
the trajectory, a phenomenon similar to sensitive dependence on initial conditions.

Yet an alternative arrangement has recently been reported in other models (Neubert, 1997; Sommerer and Ott, 1993; Ott et al., 1994; Ding and Yang, 1996; Huisman and Weissing, 1999, 2001: Vandermeeretal. [2001] This alternative suggests that even qualitative prediction may be practically unfeasible. For example, consider the simple arrangement illustrated in Eig.ـ. A one dimensional state space for a hypothetical population is illustrated with three attractors, one at zero (extinction of the population), one at the population's carrying capacity $(K)$, and one at an intermediate population density. Between any pair of attractors is a repellor that indicates the position of the separatrices $(\alpha$ and $\beta$ ) of the three basins of attraction. This is similar to the case of two alternative attractors in two basins

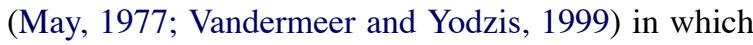
it is difficult to predict which attractor will capture a

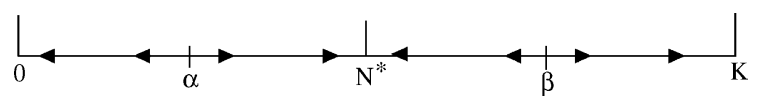

Fig. 1. State space for a simple population model in which there are tmee attractors, extinction ( 0$)$, carrying capacity $(K)$, or equilibrium $\left(N^{*}\right)$. The basin of attraction for the extinction attractor is between 0 and $\alpha$, the basin for the equilibrium is between $\alpha$ and $\beta$ and the basin for the carrying capacity is between $\beta$ and $K$. Standard interpretation of such an arrangement would suggest that a point initiated at $\alpha$ would go to either extinction or equilibrium, while a point initiated at $\beta$ would go to either equilibrium or carrying capacity.

trajectory initiated at the separatrix (e.g., a point located very near to $\alpha$ would generate a trajectory leading to either 0 or $N^{*}$, but predicting which of those two results would be increasingly difficult the closer the initiatiation point to the separatrix $\alpha$ ).

However, a surprising result emerges from some simple ecological models (Vandermeer et al., 2000). If

(a)

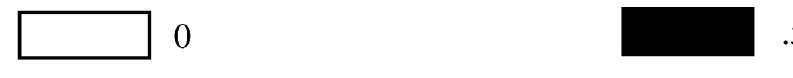

.5
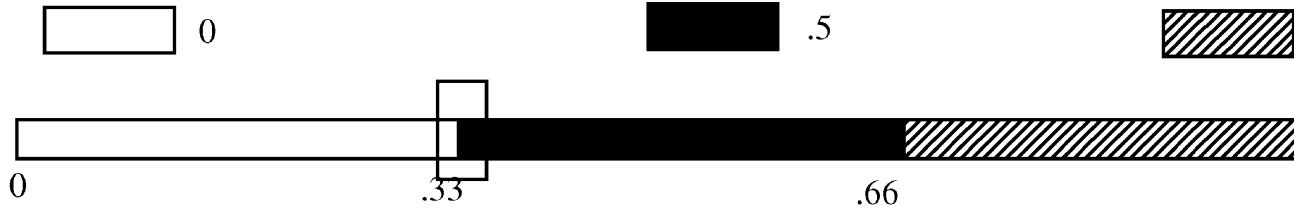

(b)

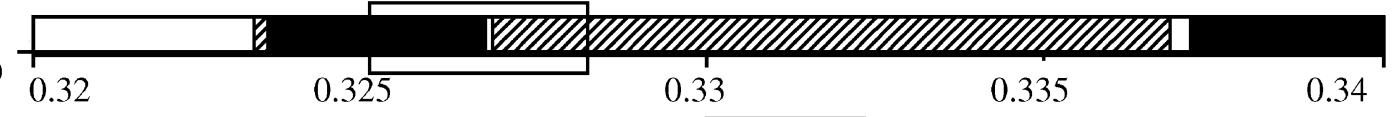

(c)

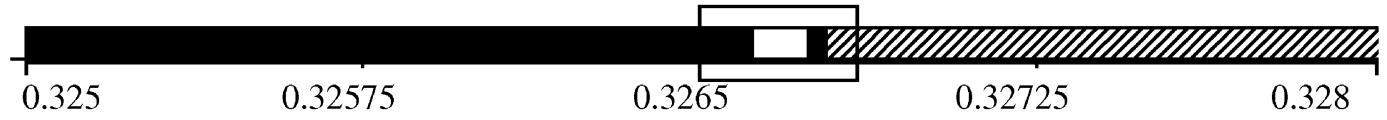

(d)

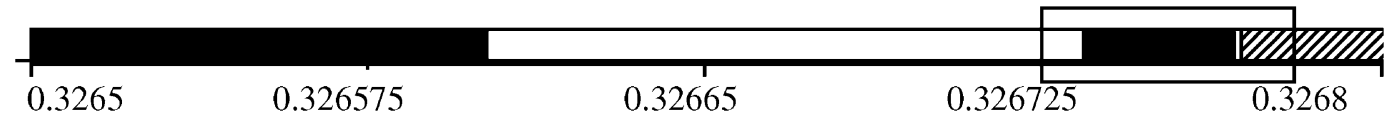

(e)

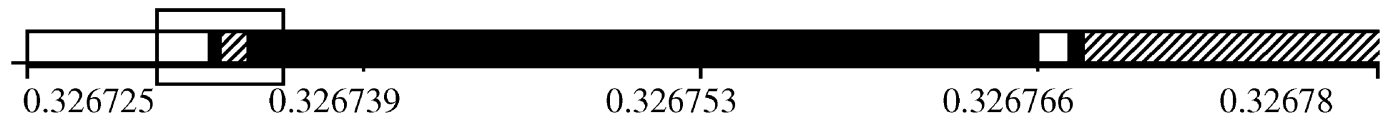

(f)

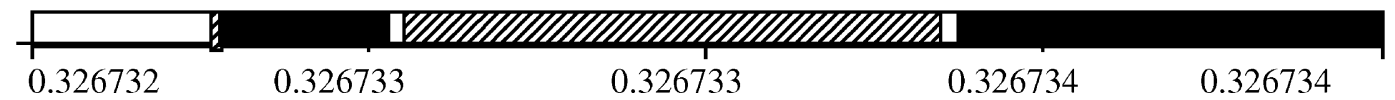

Fig. 2. Basins of attraction for a simple population model. The model is $x(t+1)=a_{i}+b_{i} x(t)$, where the values of $a_{i}$ and $b_{i}$ depend on the value of $x(0)$. When $x(t) \geq 1.0, x(t+1)=1.0$ and when $x(t) \leq 0, x(t+1)=0$. The separatrices are not points, but rather small regions with boundaries $\alpha$ and $\alpha^{\prime}, \beta$ and $\beta^{\prime}$. When $0<x(0)<\alpha, i=1$; when $\alpha<x(0)<\alpha^{\prime}, i=2$; when $\alpha^{\prime}<x(0)<\beta$, $i=3$; when $\beta<x(0)<\beta^{\prime}, i=4$; when $\beta^{\prime}<x(0)<1.0, i=5$. In the present case, $\alpha=0.33, \alpha^{\prime}=0.34, \beta=0.66, \beta^{\prime}=0.67, K=1.0$, $a_{1}=a_{5}=100, a_{2}=-51, a_{3}=-0.0625, a_{4}=-49, b_{1}=-32, b_{2}=17.85, b_{3}=0.53125, b_{4}=32.83, b_{5}=-67$. (a) Basins for the whole state space where separatrices are seen approximately at 0.33 and 0.66 ; (b) expansion of the region around the 0.33 separatrix illustrating that all three basins are contained within this region; $(\mathrm{c}-\mathrm{f})$ repeated expansion of small areas within the region of the separatrix illustrating the fractal nature of the basin boundary, including all three basins at all scales. 
the basins are multiply intersecting and fractal, which is to say they are "Wada basins" (Wusse and Yorke, [096] a trajectory initiated at either separatrix may approach any of the three attractors. This phenomenon is illustrated in Eig.2 2 for a simple piecewise linear map. As the region surrounding the separatrix is repeatedly magnified all three basins are seen to occur in what appeared to be a single point when viewed macroscopically. This multiple presence of all three basins at all scales illustrates not only that the basin boundary is fractal, but also that all three basins are interdigitated in this fractal structure. The basins are thus Wada basins (Nusse and Yorke. 1996)

The basic structure described above emerges from a variety of physical models (Nusse and Yorke. 1906) and recently in ecological models, specifically in systems that involve two or more predator prey pairs interacting with one another in some way (Vandermeer ef al, 200 The existence of such structure in nature remains to be investigated. In the following sections I first describe a simple circle map approximation to multiply coupled oscillators and illustrate how such a model may lead to multiple attractors with basins that are Wada, and second, provide a graphical interpretation of the origin of this peculiar structure.

\section{The circle map approximation to coupled oscillators}

Normally the two variables considered in predator prey dynamics are $P$, the population density (or biomass) of the predator and $V$ (victim), the population density (or biomass) of the prey. The model that has seemingly become standard for the representation of this system,

$\frac{\mathrm{d} P}{\mathrm{~d} t}=-m P+a P V /(1+b V)$

$\frac{\mathrm{d} V}{\mathrm{~d} t}=r V(1-V)-a P V /(1+b V)$

where the prey exhibits density dependence and the system is characterized by a type II functional response, with $b$ as the half-saturation constant. The parameters $a, m$ and $r$ are constants which reflect the underlying biology of the species in question.

The behavior of equations 1 has been well characterized. For purposes of the present communication, we will be concerned with the situation in which a limit cycle exists. That is, we are concerned with the cases where $2 m b<(1-b)(m+a)$. Given a limit cycle, the system can be represented as a single variable, with the following transformation,

$\Theta=\tan ^{-1}\left(\frac{C_{t}-C^{*}}{R_{t}-R^{*}}\right)$

where $C^{*}$ and $R^{*}$ refer to the average value of each of the two variables as they traverse the limit cycle (note that $C^{*}$ and $R^{*}$ will normally be near to the unstable focal point equilibrium of the system). By analyzing the transformed parameter $\Theta$, it is possible to study the qualitative behavior of the underlying system (Vandermeer. 1094: Vandermeer et al. 2000) much as is done in the study of physical oscillators (Bak. 1986: Bohr et al. 1984: Cvitanovic et al.. 1990: Jensen et al. प084) It is a simple matter to describe the general behavior of the overall system simply by knowing the value of $\Theta$. On the other hand, information on the absolute value of predator or prey populations is discarded. The focus of studies of the parameter $\Theta$ is on the qualitative behavior of the system and not on the absolute value of $P$ or $V$.

Let the basic system be forced by a periodic function (e.g. seasonality) or let it be coupled to another predator-prey system (we shall repeatedly refer to one of the systems as the forcer, even though the development here is general and includes both the situation of a true forcing function and a coupled system). We begin by examining the behavior of the system when both oscillators are completely independent of one another (i.e., begin with no coupling). Define a poincaré section as the repeated values of one of the systems (say $V_{1}, P_{1}$ ) as the other system reaches some critical value (say $V_{2 \max }$ where max refers to the peak of $V_{2}$ in its limit cycle). A trajectory cutting the poincaré section will repeatedly cut through the same point if both oscillators have exactly the same frequency. If the forcing oscillator has a period slightly greater than the other oscillator, the point at which the trajectory cuts the poincare section will not be the same at each winding, and the repeated trajectories will eventually trace out a series of points that are located on the original limit cycle of the second oscillator.

Now connect the two oscillators. Biologically this means that, for example, the predator of the second oscillator $\left(P_{2}\right)$ begins to eat the prey of the first oscilla- 
tor $\left(V_{1}\right)$; or, the two prey $\left(V_{1}\right.$ and $\left.V_{2}\right)$ begin competing with one another; or, if the first oscillator is a periodically forcing oscillator, the first oscillator may determine the value of the intrinsic rate of natural increase of the prey in the second oscillator. Many other examples of connections could be imagined. The point is that the two oscillators are coupled to one another in some way. When this happens, if the coupling is weak (below we deal with the situation of strong coupling), both oscillators will entrain, which is to say, the trajectories will eventually come to repeat themselves as if the two oscillators had the same frequency. Wherever the trajectory starts, it eventually winds up at a single trajectory, which is an equilibrium point.

Recalling the transformation of Eq. (2) Jwe now examine the behavior of $\Theta$ as the trajectory repeatedly cuts through the poincare section. If the two oscillators are completely unconnected but have identical periods, $\Theta$ will be the same each time around the first cycle. That is, if $\Theta(1)$ represents the first time around, $\Theta(2)$ the second time around, and so forth, a graph of $\Theta(t+1)$ versus $\Theta(t)$ will be simply a straight line. Thus, if we represent the dynamics of this four dimensional system as $\Theta(t+1)=f(\Theta(t))$, we have in this case, $f(\Theta(t))=\Theta(t)$. However, if the oscillators are connected, $f$ will no longer be the identity function. The simplest function that satisfies the qualitative nature of this picture is,

$\Theta(t+1)=\Theta(t)+k \sin (\Theta(t)$

where $k$ represents the degree to which the function deviates from the simple 45 degree line. $\mathbb{E q}_{\text {(3) }}$ s an example of a circle map. Scaling the map so that $\Theta$ ranges from 0 to 1 (rather than from 0 to $2 \pi$ ), we can alter $\mathrm{Eq}(3)$ o read,

$\Theta(t+1)=\Theta(t)+\left(\frac{k}{2 \pi}\right) \sin (2 \pi \Theta(t))$

In the uncoupled situation $k=0$ and Eq(4) - teverts to the simple $\Theta(t+1)=\Theta(t)$, as discussed earlier (i.e., the uncoupled situation). The parameter $k$ thus represents the strength of coupling. However, recall that $\Theta(t+1)=\Theta(t)$ represents the case in which not only is there no coupling, but the two oscillators have exactly the same period. If the period is not the same, $\Theta$ will deviate slightly each time around. Thus, the appropriate equation would be,

$\Theta(t+1)=\Omega+\Theta(t)$ where $\Omega$ is the angular difference in the point each time through the poincaré section. Combining Eq.(5) with Eq(4) we obtain,

$\Theta(t+1)=\Omega+\Theta(t)+\left(\frac{k}{2 \pi}\right) \sin (2 \pi \Theta(t))$

which is the form commonly referred to as the standard circle map. Note that this model treats the predator-prey system only from the point of view of the period of the oscillations, ignoring the amplitude. For example, in a seasonally forced predator-prey case, the value of $\Theta$ is recorded each time a constant value for the season is reached (i.e., the values of predator and prey at that point in the season are transformed with the above trigonometric transformation and thus characterized by the angle $\Theta$ ).

Now consider a system that contains more than two oscillators, i.e., there are more than two predator prey pairs or more than a single forcing function. At low levels of coupling we expect two alternative equilibrium points on the poincare section of one of the oscillating pairs, one associated with phase locking on one of the coupled systems, the other with phase locking on the other of the coupled systems. This may not always happen, since even if two of the oscillators have no direct connection with one another they are connected through the third oscillator. However, there are certain situations in which two independent oscillators have an effect on the third such that the third one will entrain on one or the other of these oscillators. For example, consider the case where predator prey system number one is affected by two others, called 0 and 2, such that system 0 receives no input from system 1 and system 2 likewise receives no input from system 1 . This could occur if, for example, the predators of systems 0 and 2 consume the prey item of system 1, but receive no benefits from such consumption. Systems 0 and 2 are thus independent of inputs from any but their own dynamics while system 1 is affected by both system 0 and 2 . Thus, at low levels of coupling, system 1 must phase lock with either system 1 or system 2 , depending on initial conditions.

In this situation the poincare section will show two attractors and two repellors. The behavior of those attractors and repellors on a map of $\Theta(t)$ versus $\Theta(t+$ 1) is illustrated in Eig . 3, where the necessary form of $f($ in $\Theta(t+1)=f(\Theta(t)))$ is obvious. 


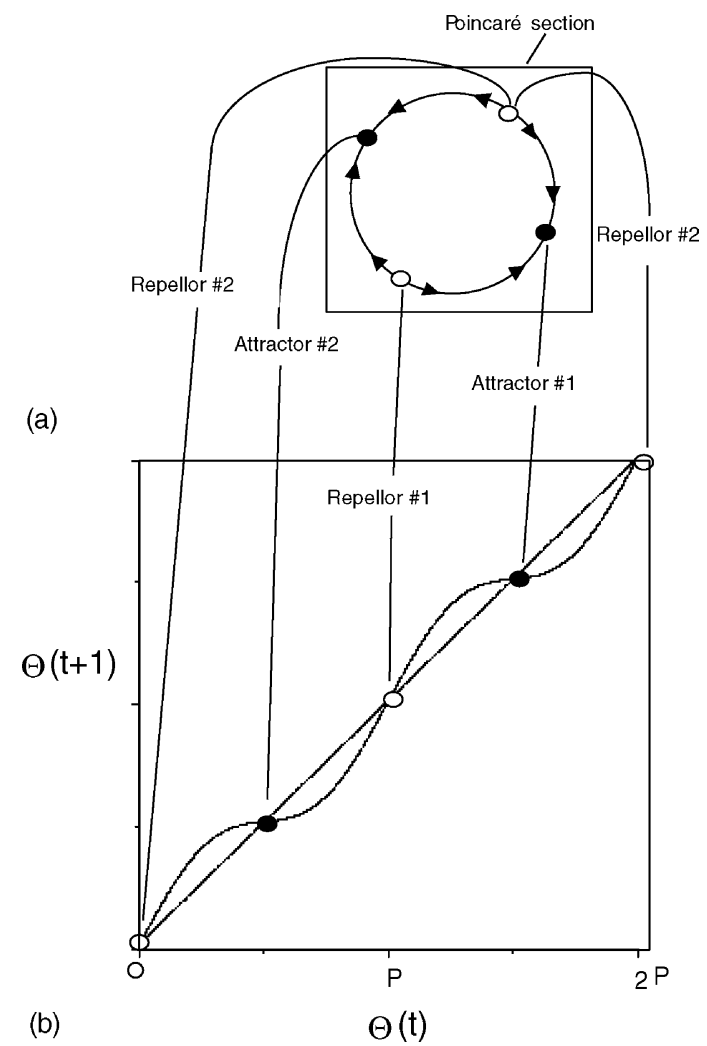

Fig. 3. Construction of the circle map from the poincare section of a forced or coupled predator prey system, where there are three coupled systems. (a) A poincaré section where there are two attractors and two repellors, each attractor represents phase locking with one pair of oscillators. The graph is of one of the predator prey pairs (predator on ordinate and prey on abcissa), and the dynamics result from the coupling to the other two oscillators and (b) circle map resulting from the poincaré section.

The simplest function corresponding to the qualitative pattern in $\mathbf{E i g} .3 \mathrm{~s}$,

$\Theta(t+1)=\Theta(t)+k \sin (b \Theta(t))$

where $b$ refers to the number of attractors in the system. Following the development of the circle map above, we can rewrite Eq(8) is,

$\Theta(t+1)=\Omega+\Theta(t)+\left(\frac{k}{2 \pi b}\right) \sin (2 \pi b \Theta(t))$

where all parameters are as defined earlier (note that the parameter $b$ appears in the denominator of the constant multiplier of the sine function-this is to retain the feature that $k=1$ represents the point of noninvertability of the system).

In the context of a larger community, $b$ represents a measure of species richness (actually the number of species, $S$, is $2 b+2$ ), while $k$ is a measure of the connectivity of the community. As an example of a larger assemblage, set $b=5, \Omega=0.5$ and $k=8$. With this parameter setting equation 1 has five distinct attractors. Initiating the system at some particular value of $\Theta$ fixes which of the five attractors will eventually be approached, but the basins of the five attractors are all interdigitated, and occupy a large fraction of the state space. This is shown in Eig. 4. Wwhere successive magnifications of sections of the state space illustrate clearly the fractal nature of the space. But unlike the other example, here there is not a seemingly single point that might be called the separatrix, but rather very large regions for which it will be in practice impossible to predict which basin will capture the trajectory. This is a level of unpredictability that goes beyond chaos, for the system illustrated in Eig.4]s not in chaos but rather has five distinct attractors (note that the state space in this example refers to the trigonometric transformation of the predator-prey ratio, as described above, and thus, each equilibrium value of $\Theta$ represents a limit cycle in the predator-prey space).

It is also worth noting that if a small stochastic force is added to $\mathbf{E q}$ (9), the system moves indeterminately around the state space in apparent chaotic motion (Fig.5) The behavior is as unpredictable as chaos yet it is not formally chaotic (the Lyapunov exponent for this example is approximately -0.066 ).

\section{Generation of Wada basins in piece-wise linear maps}

Consider the one-dimensional map, $g$, on the real line,

$$
\begin{aligned}
& g=f_{1}=\frac{b(x-a)}{0.5-a} \quad \text { for } x \leq 0.5 \\
& g=f_{2}=\frac{0.5-a(1-b)+b(x-1)}{0.5-a \quad \text { for } x>0.5}
\end{aligned}
$$

which is illustrated for the interval $0-1$ in Eig_ 6 
(a)

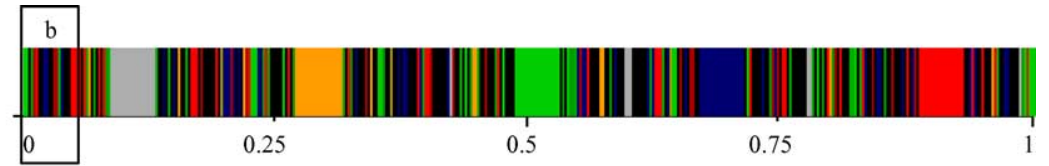

(b)

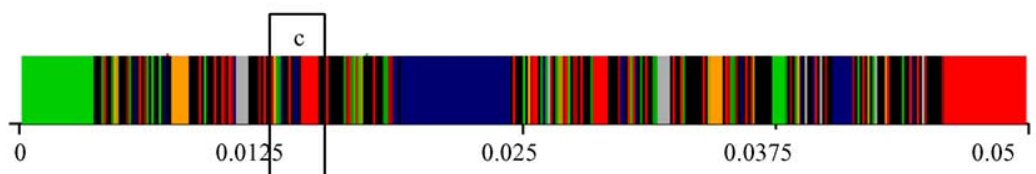

(c)

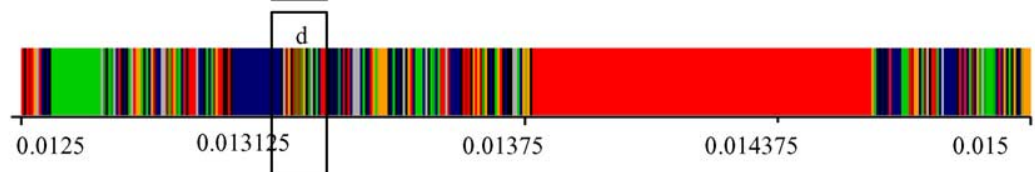

(d)

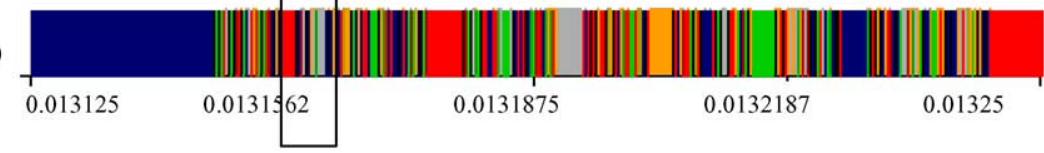

(e)

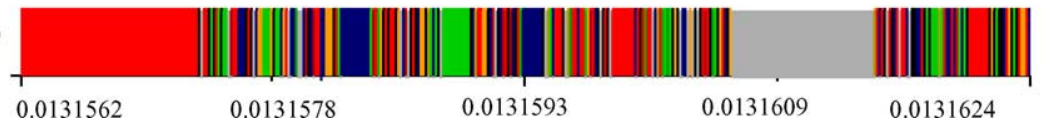

Fig. 4. Basins of attraction for the circle map with five alternative attractors. Each color signifies one of the basins. (a) The entire state space; (b) $5 \%$ of the state space (the $5 \%$ indicated by the small rectangle in a); (c-e) successively smaller $5 \%$ segments of each previous section of state space. Note the presence of all basins of attraction at all scales.

The intersection of $f_{1}$ with the 45 degree line is

$x(t+1)=\frac{a b}{a+b-0.5}$

It is clear from Eig.6 6 hat the system will remain in a chaotic state as long as,

$(1-b)>a b /(a+b-0.5)$

or

$(a / b)<(1.5-b) /(2 b-1)$

Reversing the inequality means that the system will diverge to $\pm \infty$ with the boundary between the two basins fractal.

Any interval between $(1.5-b) /(2 b-1)$ and 0.5 , will exponentially decline to zero in the limit as the inverse of $g$ is applied. To see this consider the special case of $a=0, b=1$. Thus, we have, $f_{1}=2 x$ with inverse $x=0.5 f_{1}$. The interval $\left\{x_{0}, x_{0}+\varepsilon\right\}$ will thus be $\left\{0.5 x_{0}, 0.5 x_{0}+0.5 e\right\}$ on the first reverse iteration and $\left\{0.25 x_{0}, 0.25 x_{0}+0.25 \varepsilon\right\}$ on the second iteration.
Thus, the size of the interval will be $0.5^{n} \varepsilon$ after $n$ iterations. By inspection, this behavior is obvious for any interval, except that the interval will approach one of the unstable singularities rather than zero. Thus, the origin of any interval between the two unstable singularities will be a series of intervals of exponentially decreasing size moving towards either of those two singularities.

Finally, each inverse step initiatiates a new interval, as illustrated in $\mathbb{F i g}$ And each of those new intervals initiates yet another new interval, each of which generates a cascading series of reverse iterations. And, as is clear from the graph, those iterations are multiply interdigitated, such that the area between A and B (Eig 7 7) is completely riddled with intervals that will take points through forward iterations into the first interval (which projects to the $-\infty$ basin). This graphical argument can be repeated starting with the interval that projects into the $+\infty$ basin, whence it is obvious that the entire area between A and B is riddled with intervals of all sizes that project to either of the two basins. This is an intuitive model to see how riddled or 

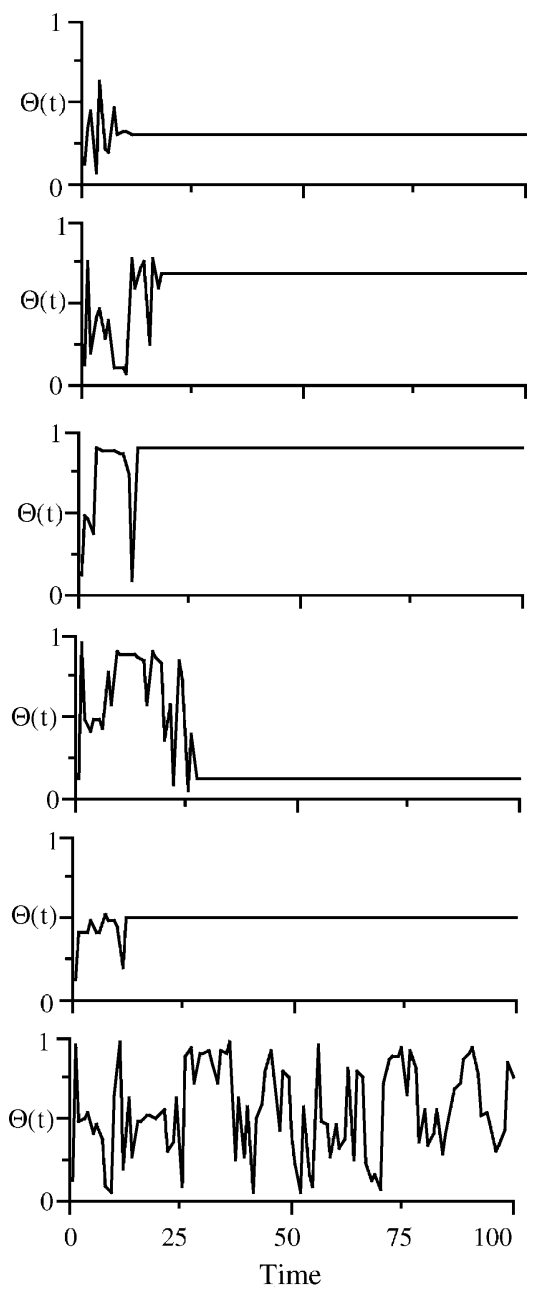

Fig. 5. Trajectories of Eq. (9) with $k=15.8, b=5$ ). Top five graphs illustrate approach to each of the five equilibrium points, after a short period of chaos-like behavior. Bottom graph illustrates behavior with an added stochastic factor (uniform random, 0-0.1), illustrating the complete chaos-like behavior. Trajectory is not chaotic (Lyapunov exponent $=-0.066$ ).

fractal basin boundaries may arise. It does not, however, correspond to the idea of a Wada basin since it only deals with two basins.

To see similar behavior leading to Wada basins, the model needs to be extended. Consider the following extended model.

$f_{1}=\frac{b(x-a)}{c-a} \quad$ for $x<c$

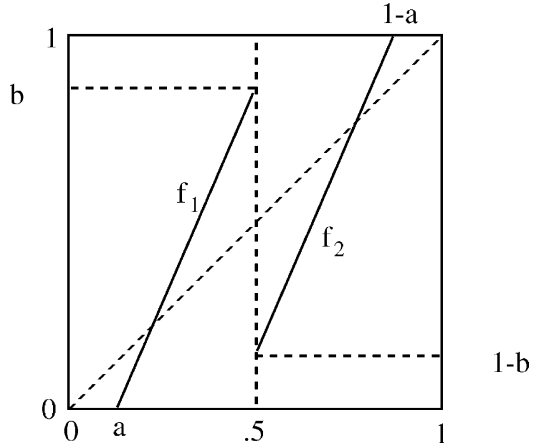

Fig. 6. The piece-wise linear map (see text).

$f_{2}=\frac{c-a(1-b)+b(x-1)}{c-a} \quad$ for $x>1-c$

$f_{3}=0.5 \quad$ for $c \leq x \leq 1-c$

which will either diverge to $\pm \infty$ or stabilize at 0.5 , depending on initial $x$. This function is illustrated in E1g.8. Jalong with several backwards projections illustrating the intermingling of all three basins. It is clear

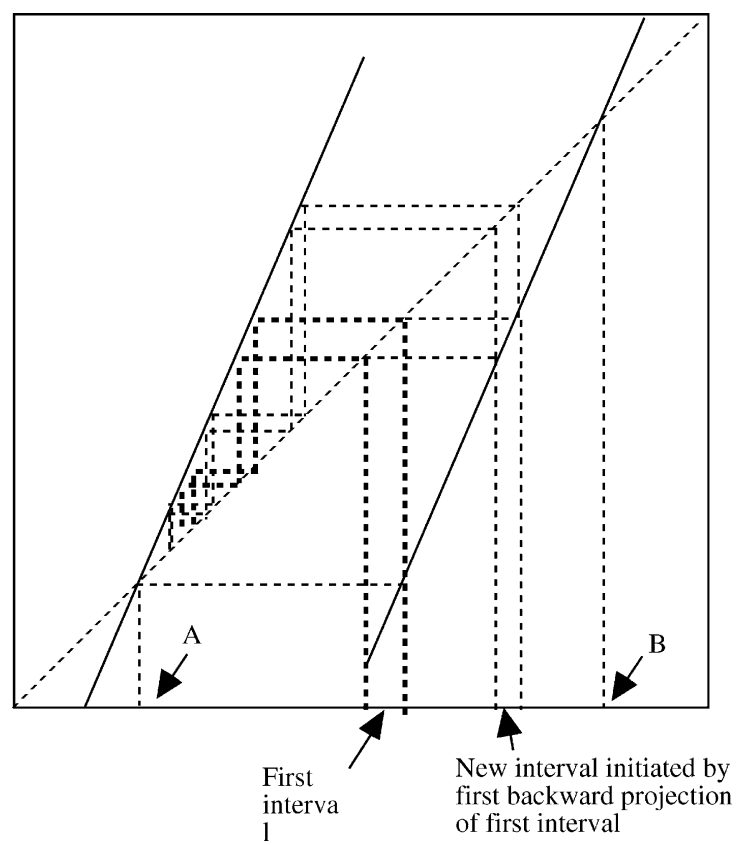

Fig. 7. Inverse projection of interval, illustrating how each initiated interval generates an exponentially decreasing interval which approaches the unstable singularity in the limit. 


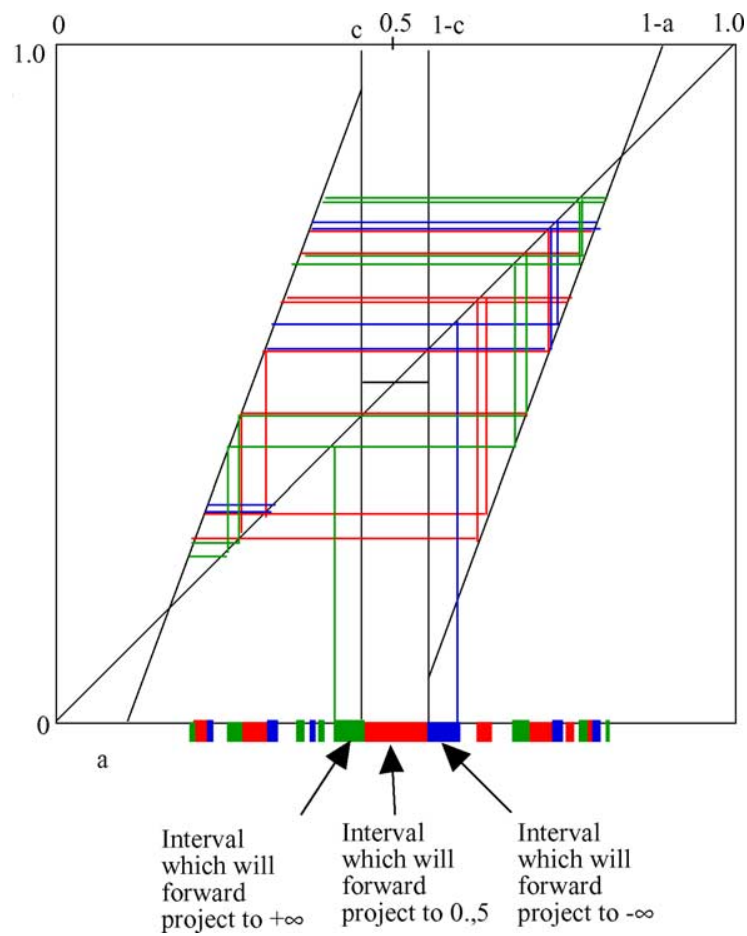

Fig. 8. Extended linear model (system 11) with three basins. The backward projections are color coded corresponding to the three basins, thus indicating on the $x$ axis to which basin the trajectory will eventually tend.

from 1 ig. 8 hat the dynamic producing intermingled basins in system 10 is the same as the dynamic in the extended model (system 11), providing a formal rendering of the origin of the three intermingled basins which thus form Wada basins.

The extended model also provides insight into the qualitative nature of Wada basins, as illustrated in E1g. Y. E1g. Ya and b depresents two different parame- ter settings for the extended model, specifically,

$b<1-a /(c-b)$

specifies case $a$ and reversing the inequality specifies case $b$. Case $c$ requires a different model which is not specified here, since the graphical interpretation of the function is the significant point. Case a represents the simple case of three alternative attractors with non-fractal basin boundaries. Case $b$ represents the case of Wada basins, the same case as analyzed in Eig. 3. Case $c$ represents what might be called a pseudowada formation, since there are still three basins of attraction, but only two of them are intermingled.

\section{Discussion}

It has been shown how simple ecosystem models can generate qualitative unpredictability above and beyond simple chaos or alternate basin structures. This phenomenon is largely the result of basin boundaries intermingling in complicated fractal, patterns, as has been noted for a variety of more realistic models (Neubert, 1997; Huisman and Weissing, 2001; Ding and Yang, 1996: Sommerer and Oft, 1993; Vandermeer et al., 200ل). It should be emphasized that, just as all models do not generate alternative states, not all models that generate alternate states have fractal basin boundaries (Cavalieri_and_Kocak, [Q99] Here it is shown how three or more basins may have complex interdigitated boundaries, such that a system seemingly perched between basins A and B may in fact converge on a limit within basin C. Such a structure is termed a Wada basin, after a legendary Mr. Wada of Japan (Alligood et al. 1996)

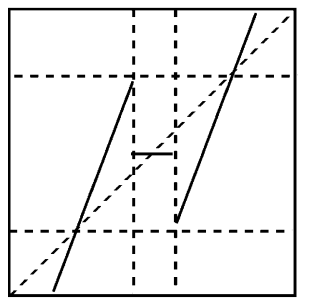

(a)

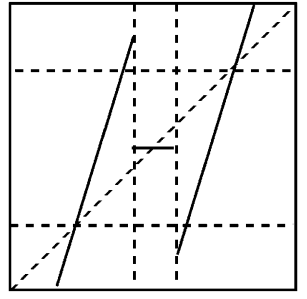

(b)

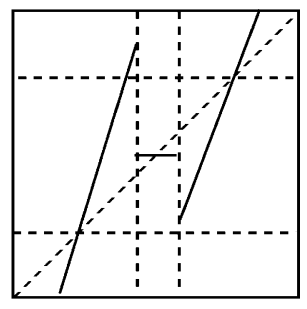

(c)

Fig. 9. The three qualitatively distinct arrangements for a piece-wise linear model of the general form as system 11 (i.e., three separate linear pieces-equations 11 cannot provide for exactly what is graphed in part c, but the qualitative behavior is nevertheless clear). 
It is worth noting that the formalism of this manuscript is thought to represent a generalization of other ecological models (e.g., Vandermeer et al. 2001) The conditions under which chaos emerges are the standard conditions normally associated with one-dimensional iterative maps, but in this case the condition on the eigenvalue is thought to represent the more complicated conditions normally associated with chaos in other, more complicated, ecological models. The important point of this paper is not the generation of chaos per se, but rather the fractal nature of the basin boundaries, which leads to a distinct form of unpredictability.

The implications of these results for ecosystem models are cautionary. For systems having Wada basins, attempts at qualitative generalization may be inherently compromised. Stipulation of alternative possibilities based on simple basin structure could be misleading if alternative basins, seemingly far removed in state space from the basins under consideration, actually exist and share fractal boundaries with them. For example the seasonally forced Lotka Volterra system has one parameter combination that results in three alternative limit cycle attractors with three distinct oscillatory patterns Dandermeer et al. 2001 It may be of interest for an ecosystem manager to predict which of the three patterns will exist for a given time period. Knowing that the present condition is somewhere between pattern $\mathrm{A}$ and pattern $\mathrm{B}$ does not allow the manager to conclude that pattern $\mathrm{C}$ is unlikely to occur. Thus, even the qualitative prediction of "either pattern A or B" is not strictly possible if the current state is near any of the basin boundaries.

This phenomenon must be added to the now well-known ideas of alternative equilibria May. [Q77) chaos (May, 1976), as influencing the ability to predict ecosystem behavior. While these previous cautionary tales emphasized the impossibility of making quantitative predictions, the existence of Wada basins suggests something more troublesome. It may have been difficult to predict which of two equilibria would be approached when starting on the border of the two basins (May, पा and the boundaries between those two basins may become large and riddled (Deubert, 297: Huisman and Weissing, 2001) but it had always been tacitly assumed one or the other of the two possibilities would ultimately capture the trajectory. The results here suggest that even though a system is perched on the separatrix between two alternative states, it may in fact wind up in a third (or fourth or fifth) state, and unpredictably so.

\section{Acknowledgements}

Jim Koopman, Franco Nari, Peter Yodzis and Mary Anne Evans provided valuable comments on earlier versions of this manuscript.

\section{References}

Alligood, K.T., Sauer, T.D., Yorke, J.A., 1996. Chaos: An Introduction to Dynamical Systems. Springer, Berlin.

Bak, P., 1986. The devil's staircase. Phys. Today 39-45.

Bohr, T., Bak, P., Jensen, M.H., 1984. Transition to chaos by interaction of resonances in dissipative systems. II. Josephson junctions, charge-density waves, and standard maps. Phys. Rev. A 30, 1970-1981.

Cavalieri, L.F., Koçak, H., 1999. Coexistence of multipule attractors and its consequence for a three species food chain. In: Hawkins, B.A., Howard, V.C. (Eds.), Theoretical Approaches to Biological Control. Cambridge University Press, Cambridge, UK.

Cvitanovic, P., Gunaratne, G.H., Vinson, M.J., 1990. On the mode-locking universality for critical circle maps. Nonlinearity 3, 873-885.

Ding, M., Yang, W., 1996. Observation of intermingled basins in coupled oscillators exhibiting synchronized chaos. Phys. Rev. E 54, 2489-2494.

Hastings, A., Hom, C.L., Ellner, S., Turchin, P., Godfray, H.C.J., 1993. Chaos in ecology: is mother nature a strange attractor? Ann. Rev. Ecol. System. 24, 1-33.

Huisman, J., Weissing, F.J., 1999. Biodiversity of plankton by species oscillations and chaos. Nature 401, 407-410.

Huisman, J., Weissing, F.J., 2001. Fundamental unpredictability in multispecies competition. Am. Nat. 157, 488-494.

Jensen, M.H., Bak, P., Bohr, T., 1984. Transition to chaos by interaction of resonances in dissipative systems. I. Circle maps. Phys. Rev. A 30, 1960-1969.

Levins, R., 1966. The strategy of model building in population biology. Am. Scientist 54, 421-431.

May, R.M., 1973. Stability and complexity in model ecosystems. Monographs in Population Biology, No. 6. Princeton University Press, Princeton, N.J.

May, R.M., 1976. Simple mathematical models with very complicated dynamics. Nature 261, 459-467.

May, R.M., 1977. Thresholds and breakpoints in ecosystems with a multiplicity of stable states. Nature 269, 471477.

Neubert, M.G., 1997. A simple population model with qualitatively uncertain dynamics. J. Theor. Biol. 189, 399-411.

Nusse, H.E., Yorke, J.A., 1996. Basins of attraction. Science 271, $1376-1380$. 
Ott, E.J., Alexander, C., Kan, I., Sommerer, J.C., Yorke, J.A., 1994. The transition to chaotic attractors with riddled basins. Phys. D 76, 384-410.

Sommerer, J.C., Ott, E., 1993. A physical system with qualitatively uncertain dynamics. Nature 365, 138-140.

Vandermeer, J.H., 1994. The qualitative behavior of coupled predator prey oscillations as deduced from simple circle maps. Ecol. Model. 73, 135-148.
Vandermeer, J.H., Yodzis, P., 1999. Basin boundary collision as a model of discontinuous change in ecosystems. Ecology 80, $1817-1827$.

Vandermeer, J.H., Stone, L., Blasius, B., 2001. Categories of chaos and fractal basin boundaries in a forced Lotka-Volterra model. Chaos, Solitons and Fractals 12, 265276. 